\title{
Arm Recoil Result
}

National Cancer Institute

\section{Source}

National Cancer Institute. Arm Recoil Result. NCI Thesaurus. Code C87063.

The result obtained from a test commonly administered to assess the physiologic response to extending the arms as straight as possible alongside the body when lying down. 